\title{
DECENTRALIZED FUZZY-INTEGRAL-SLIDING CONTROL FOR A CLASS OF TITO UNCERTAIN NONLINEAR SYSTEMS WITH APPLICATION TO A 2-DOF HELICOPTER MODEL
}

\author{
Kazem Zare, Hamid Reza Koofigar \\ University of Isfahan, Department of Electrical Engineering, Isfahan, Iran \\ e-mail:k.zare@eng.ui.ac.ir; koofigar@eng.ui.ac.ir
}

\begin{abstract}
In this paper, a novel fuzzy-integral-sliding controller (FISC) is designed for coupled nonlinear two-input two-output (TITO) systems. Decomposing the original system into two subsystems, the coupling effects are modelled as uncertainties. In order to ensure the robustness properties with respect to system uncertainties and external disturbances, the sliding mode technique with a proportional integral (PI) sliding surface is adopted. On the other hand, to avoid the chattering phenomenon, the Takagi-Sugeno fuzzy rules are incorporated into the control algorithm, which forms a fuzzy sliding controller. The stability analysis is also presented based on the Lyapunov stability theorem. The proposed FISC is then applied to control the elevation and azimuth angles of Humusoft CE150, as a two degree of freedom (DOF) laboratory helicopter model with highly cross-coupled dynamics. The simulation results are also presented to demonstrate the performance of the proposed control scheme.
\end{abstract}

Keywords: CE150 helicopter, non-linear control, FISC, robustness, decoupling

\section{Introduction}

In many multi-input-multi-output (MIMO) systems, decentralized control is adopted instead of multivariable centralized control, since it is simple to design and easy to tune, implement and maintain (Chiu and Arkun, 1990). Besides, the centralized multi-loop controllers cannot be tuned independently due to interactions. Removing such a drawback, the so-called detuning method (Chien et al., 1999) suggests to design each loop, based on the corresponding diagonal element and ignore the interactions from other loops. Then, the controllers are detuned considering the interactions until some prescribed limit is attained. The detuning methods are simple, but the closed loop stability is not necessarily ensured.

TITO systems are one of the main categories of multivariable systems, with non-negligible input-output interactions (Jevtović and Mataušek, 2010; Tavakoli et al., 2006). Jevtović and Mataušek (2010) presented a decentralized controller design method with an ideal decoupler. Sliding-mode control (SMC) has been extensively used to ensure the robustness properties against the system uncertainties (Huang et al., 2003; Utkin, 1992). Also, it has been applied to reduce the sensitivities to parameter variations (Hung et al., 1993; Utkin, 1977). More recently, fuzzy techniques together with the sliding-mode control have been applied to uncertain nonlinear systems, which can overcome the chattering phenomenon (Glower and Munighan, 1997; Kim and Lee, 1995). Such systems as robots, two-stage vibration isolation systems (Zhao et al., 2007), TRMS, and CE150 helicopters are some benchmarks for evaluating the performance of the previous works. The proportional-integral controller, using Q-learning and PSO algorithms, has been used to control the azimuth and elevation angles of a helicopter, without taking the coupling into account (Boubertakh et al., 2010, 2012). Removing such a drawback, fuzzy logic together with PID controller has been adopted (Velagic and Osmic, 2010). However, it is not 
straightforward to form the fuzzy-rule base and the experts knowledge is the main design factor (Sapiński, 2005; Zorić et al., 2013).

In this paper, a decentralized FISC is proposed for a class of uncertain interconnected TITO systems. The mathematical model of TITO systems is first decoupled into two subsystems, and the cross-coupling effects between subsystems are considered as system uncertainty. An adaptive identifier is introduced for unknown parameter estimation. Then, in order to remove the offset and ensure the asymptotic stability, a proportional integral (PI) sliding surface is adopted for two subsystems, and Takagi-Sugeno fuzzy rules are incorporated into the control algorithm to avoid the chattering phenomenon. The developed algorithm is applied to the CE150 helicopter as a highly cross-coupled two input-two output laboratory system (Humusoft, 1989). The dynamical model is decoupled into a horizontal subsystem (HS) and a vertical subsystem (VS). The cross-coupling effect between the main rotor and the tail rotor is considered as the uncertainty. Simulation results illustrate the tracking performance in the presence of model uncertainties and external disturbance. In general, compared with some previous works, the proposed FISC technique for TITO systems has some benefits as (i) simplicity and universality, (ii) removing the chattering phenomenon of conventional SMC, (iii) ensuring robustness against uncertainties and disturbances.

This paper is organized as follows. The control problem of a TITO nonlinear coupled system based on unknown parameter identification is described in Section 2. In Section 3, the design procedure of fuzzy-integral-sliding controllers for subsystems and the stability analysis are presented. Introducing the mathematical model of the CE150 helicopter, the proposed control algorithms are applied and the simulation results are demonstrated in Section 4. Finally, the concluding remarks are given in Section 5.

\section{Problem formulation}

Consider a class of TITO nonlinear coupled systems, expressed as

$$
\dot{\mathbf{X}}=f(\mathbf{X})+g(\mathbf{X}) U+\mathbf{d}(t) \quad \mathbf{Y}=C \mathbf{X}
$$

where $\mathbf{X}=\left[x_{1}, x_{2}, \ldots, x_{n}\right]^{\mathrm{T}} \in R^{n}$ denotes the state vector, $U, Y \in R^{2}$ represent the control input and system output respectively, $f(\mathbf{X})$ and $g(\mathbf{X})$ contain some nonlinear functions and $\mathbf{d}(t)$ is the bounded external disturbance. To facilitate the designing of the controller, TITO model (2.1) is pseudo-decomposed into an HS and a VS as

$$
\begin{array}{ll}
\dot{\mathbf{X}}_{h}=f_{h}\left(\mathbf{X}_{h}\right)+g_{h}(\mathbf{X}) U+\mathbf{d}_{1} & h=1,2, \ldots, k \\
\dot{\mathbf{X}}_{v}=f_{v}\left(\mathbf{X}_{v}\right)+g_{v}(\mathbf{X}) U+\mathbf{d}_{2} & v=k+1, k+2, \ldots, n \\
\mathbf{Y}=\left[y_{h}, y_{v}\right]^{\mathrm{T}}=\left[x_{1}, x_{k+1}\right]^{\mathrm{T}} &
\end{array}
$$

where $\mathbf{X}=\left[\mathbf{X}_{h}, \mathbf{X}_{v}\right]^{\mathrm{T}}$. Using the Jacobian, system dynamics (2.2) can be interpreted as two linear subsystems perturbed by model uncertainties $\Delta \mathbf{F}_{h}, \Delta \mathbf{F}_{v}$ and disturbances $\mathbf{d}_{1}$ and $\mathbf{d}_{2}$, as

$$
\dot{\mathbf{X}}_{h}=\mathbf{A}_{h} \mathbf{X}_{h}+\mathbf{B}_{h} u_{1}+\Delta \mathbf{F}_{h}+\mathbf{d}_{1} \quad \dot{\mathbf{X}}_{v}=\mathbf{A}_{v} \mathbf{X}_{v}+\mathbf{B}_{v} u_{2}+\Delta \mathbf{F}_{v}+\mathbf{d}_{2}
$$

where

$$
\mathbf{A}_{h}=\left[\begin{array}{ccc}
\frac{\partial f_{1}}{\partial x_{1}} & \cdots & \frac{\partial f_{1}}{\partial x_{k}} \\
\vdots & \ddots & \vdots \\
\frac{\partial f_{k}}{\partial x_{1}} & \cdots & \frac{\partial f_{k}}{\partial x_{k}}
\end{array}\right] \quad \mathbf{A}_{v}=\left[\begin{array}{ccc}
\frac{\partial f_{k+1}}{\partial x_{k+1}} & \cdots & \frac{\partial f_{k+1}}{\partial x_{n}} \\
\vdots & \ddots & \vdots \\
\frac{\partial f_{n}}{\partial x_{k+1}} & \cdots & \frac{\partial f_{n}}{\partial x_{n}}
\end{array}\right]
$$




$$
\mathbf{B}_{h}=\left[\frac{\partial g_{1}}{\partial u_{1}}, \ldots, \frac{\partial g_{k}}{\partial u_{1}}\right]^{\top} \quad \mathbf{B}_{v}=\left[\frac{\partial g_{k+1}}{\partial u_{2}}, \ldots, \frac{\partial g_{n}}{\partial u_{2}}\right]^{\mathrm{T}}
$$

Two types of adaptive parameter identifiers, including the parallel model, and a seriesparallel model may be adopted to identify the unknown matrices of linear systems (Ioannou and Sun, 1995). In this paper, the unknown parameters of $\mathbf{A}_{h}$ and $\mathbf{A}_{v}$ are estimated, based on a series-parallel model, defined as

$$
\begin{aligned}
& \dot{\hat{\mathbf{X}}}_{h}=\mathbf{A}_{m h} \widehat{\mathbf{X}}_{h}+\left(\widehat{\mathbf{A}}_{h}-\mathbf{A}_{m h}\right) \mathbf{X}_{h}+\mathbf{B}_{h} u_{1} \\
& \dot{\hat{\mathbf{X}}}_{v}=\mathbf{A}_{m v} \widehat{\mathbf{X}}_{v}+\left(\widehat{\mathbf{A}}_{v}-\mathbf{A}_{m v}\right) \mathbf{X}_{v}+\mathbf{B}_{v} u_{2}
\end{aligned}
$$

where $\widehat{\mathbf{A}}_{h}$ and $\widehat{\mathbf{A}}_{v}$ are the estimates of $\mathbf{A}_{h}$ and $\mathbf{A}_{v}$, respectively, $\mathbf{A}_{m h}$ and $\mathbf{A}_{m v}$ are two arbitrary stable matrices, and $\widehat{\mathbf{X}}_{h}$ and $\widehat{\mathbf{X}}_{v}$ denote respectively the estimates of $\mathbf{X}_{h}$ and $\mathbf{X}_{v}$.

Define the estimation errors

$$
\mathbf{e}_{h}=\mathbf{X}_{h}-\widehat{\mathbf{X}}_{h} \quad \mathbf{e}_{v}=\mathbf{X}_{v}-\widehat{\mathbf{X}}_{v}
$$

whose dynamics are

$$
\dot{\mathbf{e}}_{h}=\mathbf{A}_{m h} \mathbf{e}_{h}-\widetilde{\mathbf{A}}_{h} \mathbf{X}_{h} \quad \dot{\mathbf{e}}_{v}=\mathbf{A}_{m v} \mathbf{e}_{v}-\widetilde{\mathbf{A}}_{v} \mathbf{X}_{v}
$$

where $\widetilde{\mathbf{A}}_{h}=\widehat{\mathbf{A}}_{h}-\mathbf{A}_{h}$ and $\widetilde{\mathbf{A}}_{v}=\widehat{\mathbf{A}}_{v}-\mathbf{A}_{v}$ represent the matrix estimation errors. Ignoring the index of the symbols, choose a Lyapunov function candidate as

$$
V(\mathbf{e}, \widetilde{\mathbf{A}})=\mathbf{e}^{\mathrm{T}} \mathbf{P e}+\operatorname{tr}\left(\frac{1}{\gamma} \widetilde{\mathbf{A}}^{\mathrm{T}} \mathbf{P} \widetilde{\mathbf{A}}\right)
$$

where $\gamma$ is a positive constant and $\mathbf{P}=\mathbf{P}^{\mathrm{T}}>\mathbf{0}$ is chosen as a solution of

$$
\mathbf{P} \mathbf{A}_{m}+\mathbf{A}_{m}^{\mathrm{T}} \mathbf{P}=-\mathbf{Q}
$$

The time derivative of $\mathrm{V}$ is calculated as

$$
\dot{V}=\dot{\mathbf{e}}^{\mathrm{T}} \mathbf{P e}+\mathbf{e}^{\mathrm{T}} \mathbf{P} \dot{\mathbf{e}}+\operatorname{tr}\left(\frac{1}{\gamma} \dot{\tilde{\mathbf{A}}}^{\mathrm{T}} \mathbf{P} \widetilde{\mathbf{A}}+\frac{1}{\gamma} \widetilde{\mathbf{A}}^{\mathrm{T}} \mathbf{P} \dot{\tilde{\mathbf{A}}}\right)
$$

By incorporating error dynamics (2.6) and equality (2.7), one obtains

$$
\begin{aligned}
\dot{V} & =\left(\mathbf{e}^{\mathrm{T}} \mathbf{A}_{m}^{\mathrm{T}}-\mathbf{X}^{\mathrm{T}} \widetilde{\mathbf{A}}^{\mathrm{T}}\right) \mathbf{P e}+\mathbf{e}^{\mathrm{T}} \mathbf{P}\left(\mathbf{A}_{m} \mathbf{e}-\widetilde{\mathbf{A}} \mathbf{X}\right)+2 \operatorname{tr}\left(\frac{1}{\gamma} \widetilde{\mathbf{A}}^{\mathrm{T}} \mathbf{P} \dot{\tilde{\mathbf{A}}}\right) \\
& =-\mathbf{e}^{\mathrm{T}} \mathbf{Q} \mathbf{e}+2 \operatorname{tr}\left(\left(\frac{1}{\gamma} \widetilde{\mathbf{A}}^{\mathrm{T}} \mathbf{P} \dot{\widetilde{\mathbf{A}}}-\widetilde{\mathbf{A}}^{\mathrm{T}} \mathbf{P} \mathbf{e} \mathbf{X}^{\mathrm{T}}\right)\right.
\end{aligned}
$$

In order to obtain $\dot{V}=-\mathbf{e}^{\mathrm{T}} \mathbf{Q} \mathbf{e}<0$, choose $\dot{\widetilde{\mathbf{A}}}=\gamma \mathbf{e} \mathbf{X}^{\mathrm{T}}$. Hence, $\dot{V}$ is negative definite, i.e., the stability is ensured. This implies that the adaptation mechanisms for $\widehat{\mathbf{A}}_{h}$ and $\widehat{\mathbf{A}}_{v}$ are

$$
\dot{\widehat{\mathbf{A}}}_{h}=\gamma_{h} \mathbf{e}_{h} \mathbf{X}_{h}^{\mathrm{T}} \quad \dot{\widehat{\mathbf{A}}}_{v}=\gamma_{v} \mathbf{e}_{v} \mathbf{X}_{v}^{\mathrm{T}}
$$

where $\gamma_{h}, \gamma_{v}>0$.

Now, equations in (2.3), with incorporating the estimations $\widehat{\mathbf{A}}_{h}$ and $\widehat{\mathbf{A}}_{v}$, can be rewritten as

$$
\dot{\mathbf{X}}_{h}=\widehat{\mathbf{A}}_{h} \mathbf{X}_{h}+\mathbf{B}_{h} u_{1}+\Delta \mathbf{F}_{h}+\mathbf{d}_{1} \quad \dot{\mathbf{X}}_{v}=\widehat{\mathbf{A}}_{v} \mathbf{X}_{v}+\mathbf{B}_{v} u_{2}+\Delta \mathbf{F}_{v}+\mathbf{d}_{2}
$$


Letting the desired reference vectors of two subsystems be $\mathbf{X}_{d h}$ and $\mathbf{X}_{d v}$, the error vectors are defined as

$$
\mathbf{E}_{h}=\mathbf{X}_{h}-\mathbf{X}_{d h} \quad \mathbf{E}_{v}=\mathbf{X}_{v}-\mathbf{X}_{d v}
$$

which yields the error dynamics

$$
\begin{aligned}
\dot{\mathbf{E}}_{h} & =\widehat{\mathbf{A}}_{h} \mathbf{E}_{h}+\mathbf{B}_{h} u_{1}-\dot{\mathbf{X}}_{d h}+\widehat{\mathbf{A}}_{h} \mathbf{X}_{d h}+\Delta \mathbf{F}_{h}+\mathbf{d}_{1} \\
\dot{\mathbf{E}}_{v} & =\widehat{\mathbf{A}}_{v} \mathbf{E}_{v}+\mathbf{B}_{v} u_{2}-\dot{\mathbf{X}}_{d v}+\widehat{\mathbf{A}}_{v} \mathbf{X}_{d v}+\Delta \mathbf{F}_{v}+\mathbf{d}_{2}
\end{aligned}
$$

Due to the presence of model uncertainties and disturbances in (2.14), the fuzzy integral sliding surface controllers are designed to make the tracking error converge to zero.

\section{Fuzzy sliding controller design}

Both error dynamics in (2.14) can be described as

$$
\dot{\mathbf{E}}=\widehat{\mathbf{A}} \mathbf{E}+\mathbf{B} u-\dot{\mathbf{X}}_{d}+\widehat{\mathbf{A}} \mathbf{X}_{d}+\Delta \mathbf{F}+\mathbf{d}
$$

In order to eliminate the steady state tracking error of the system, a PI sliding surface is designed for each subsystem. To this end, take the switching surface as

$$
\sigma=\boldsymbol{\alpha}_{1} \mathbf{E}+\boldsymbol{\alpha}_{2} \int \mathbf{E} d t
$$

whee $\boldsymbol{\alpha}_{1}, \boldsymbol{\alpha}_{2}$ are two constant vectors. Differentiating (3.2) along error trajectory (3.1), yields

$$
\dot{\sigma}=\boldsymbol{\alpha}_{1}\left(\widehat{\mathbf{A}} \mathbf{E}+\mathbf{B} u-\dot{\mathbf{X}}_{d}+\widehat{\mathbf{A}} \mathbf{X}_{d}+\Delta \mathbf{F}+\mathbf{d}\right)+\boldsymbol{\alpha}_{2} \mathbf{E}
$$

In order to derive a control law to ensure the closed loop robust stability, choose the Lyapunov function

$$
V(x)=\frac{1}{2} \sigma^{2}
$$

Taking the time derivative of (3.4) and incorporating (3.3), one can obtain

$$
\dot{V}=\sigma \dot{\sigma}=\sigma\left[\boldsymbol{\alpha}_{1}\left(\widehat{\mathbf{A}} \mathbf{E}+\mathbf{B} u-\dot{\mathbf{X}}_{d}+\widehat{\mathbf{A}} \mathbf{X}_{d}+\Delta \mathbf{F}+\mathbf{d}\right)+\boldsymbol{\alpha}_{2} \mathbf{E}\right]
$$

To ensure the closed-loop stability, based on the Lyapunov stability theorem, the control input is determined such that $\dot{V}$ is negative definite, i.e.

$$
\dot{V} \leqslant-\eta|\sigma|
$$

where $\eta$ is a positive constant. The control input $u$ is chosen as

$$
u=u_{e q}-\left(\boldsymbol{\alpha}_{1} \mathbf{B}\right)^{-1} K \operatorname{sgn}(\sigma)
$$

where $\boldsymbol{\alpha}_{1} \mathbf{B}$ is non-singular, $K$ is a positive parameter to be designed, sgn represents the sign function and the equivalent control action $u_{e q}$ is obtained by solving $\dot{\sigma}=0$ in (3.3), in the absence of uncertainties and disturbances, i.e.

$$
u_{e q} \equiv-\left(\boldsymbol{\alpha}_{1} \mathbf{B}\right)^{-1}\left(\boldsymbol{\alpha}_{1} \widehat{\mathbf{A}} \mathbf{E}+\boldsymbol{\alpha}_{2} \mathbf{E}-\boldsymbol{\alpha}_{1} \dot{\mathbf{X}}_{d}+\boldsymbol{\alpha}_{1} \widehat{\mathbf{A}} \mathbf{X}_{d}\right)
$$


Now, replace control input (3.7) into (3.5) to obtain

$$
\dot{V}=\sigma\left[\boldsymbol{\alpha}_{1}(\Delta \mathbf{F}+\mathbf{d})-K \operatorname{sgn}(\sigma)\right]
$$

In order to satisfy (3.6), it is sufficient to choose $K \geqslant\left|\eta+\boldsymbol{\alpha}_{1}(\Delta \mathbf{F}+\mathbf{d})\right|$, but $\Delta \mathbf{F}$ and $\mathbf{d}$ are unknown. Moreover, the chattering phenomenon is inevitable due to the existence of the sign function in control input $u$. Removing such drawbacks, the fuzzy switching mechanism $u_{f s}$ is incorporated into (3.7) to form

$$
u=-\left(\boldsymbol{\alpha}_{1} \mathbf{B}\right)^{-1}\left(\boldsymbol{\alpha}_{1} \widehat{\mathbf{A}} \mathbf{E}+\boldsymbol{\alpha}_{2} \mathbf{E}-\boldsymbol{\alpha}_{1} \dot{\mathbf{X}}_{d}+\boldsymbol{\alpha}_{1} \widehat{\mathbf{A}} \mathbf{X}_{d}-u_{f s}\right)
$$

Letting $\sigma$ and $u_{f s}$ be the input and the output variables of the FISC, respectively, the instinctive rules can be written as

$$
\begin{array}{lll}
u_{f s}>0 & \text { if } & \sigma<0 \\
u_{f s}=0 & \text { if } & \sigma=0 \\
u_{f s}<0 & \text { if } & \sigma>0
\end{array}
$$

The input and output spaces are fuzzily partitioned into three fuzzy sets. The input and output membership functions are shown in Fig. 1.

(a)

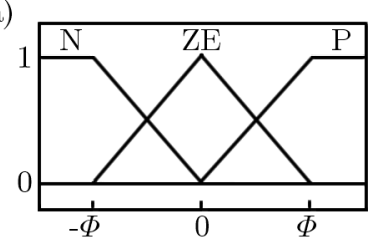

(b)

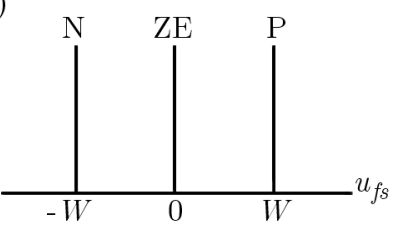

Fig. 1. Membership functions of FISC for (a) input, (b) output

The labels for the input and output membership functions are: N (negative), ZE (zero), and $\mathrm{P}$ (positive), with $\Phi$ and $W$ as two real constants. As summarized in Table 1, the $i$-th fuzzy rule for the system takes form

$$
R_{i}: \text { IF } \sigma \text { is } F_{\sigma}^{i} \text { THEN } u_{f s} \text { is } F_{u_{f s}}^{i} \quad i=1,2,3
$$

where $F_{\sigma_{h}}^{i}$ and $F_{u_{f s h}}^{i}$ are the corresponding fuzzy sets, defined based on (3.11).

Table 1. Fuzzy-rule base for FISC

\begin{tabular}{|l|lll|}
\hline$(\sigma)$ input & $\mathrm{N}$ & $\mathrm{ZE}$ & $\mathrm{P}$ \\
\hline$\left(u_{f s}\right)$ output & $\mathrm{P}$ & $\mathrm{ZE}$ & $\mathrm{N}$ \\
\hline
\end{tabular}

By using the centroid defuzzification technique, the switching input $u_{f s}$ is calculated. The general framework of the TITO control system with the FISC is presented in Fig. 2.

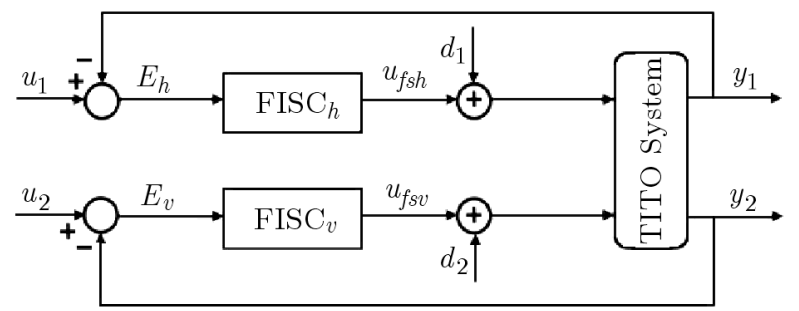

Fig. 2. Decentralized FISC system 


\section{Application of FISC to helicopter control}

The schematic of a CE150 helicopter system is shown in Fig. 3.

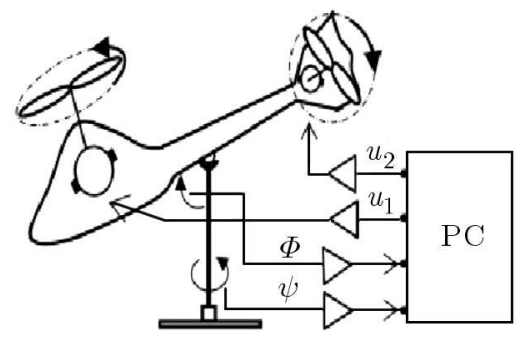

Fig. 3. Helicopter configuration (Boubertakh et al., 2012)

\subsection{Helicopter model}

Humusoft CE150, as a 2 DOF laboratory helicopter (Humusoft, 1989) consists of a body with two DC motors which drive the propellers. The axes of the body rotation are perpendicular as well as the axes of the motors. The both body position angles, i.e. azimuth angle $\psi$ in the horizontal plane and elevation angle $\Phi$ in the vertical one are influenced by the rotating propellers simultaneously. The mathematical model is formed with two manipulated inputs $u_{1}$ and $u_{2}$ and two measured outputs $(\Phi, \psi)$. The user of the simulator communicates with the system via the data-processing interface, the entries $u_{1}$ and $u_{2}$ and the outputs, scaled in the interval $[-1,+1]$, where " 1 " is called the Machine Unit [MU]. The mathematical model of the helicopter is given by a set of differential equations as (Dutka et al., 2003; Morbidi, 2006/2007)

$$
\begin{aligned}
& \dot{x}_{1}=x_{2} \quad \dot{x}_{2}=\frac{1}{I_{\psi}}\left(-\sin \left(x_{1}\right) \tau_{g}-x_{2} b_{\psi}+a_{1}\left(x_{3}\right)^{2}+b_{1} x_{3}-k_{\text {gyro }} \cos \left(x_{1}\right) x_{6} u_{1}\right) \\
& \dot{x}_{3}=-\frac{1}{T_{1}} x_{3}+\frac{1}{T_{1}} x_{4} \quad \dot{x}_{4}=-\frac{1}{T_{1}} x_{4}+\frac{1}{T_{1}} u_{1} \quad \dot{x}_{5}=x_{6} \\
& \dot{x}_{6}=\frac{1}{I_{\varphi}}\left(-x_{6} b_{\varphi}+a_{2}\left(x_{7}\right)^{2}+b_{2} x_{7}-x_{9}-\frac{k_{r} t_{o r}}{t_{p r}} u_{1}\right) \quad \dot{x}_{7}=-\frac{1}{T_{2}} x_{7}+\frac{1}{T_{2}} x_{8} \\
& \dot{x}_{8}=-\frac{1}{T_{2}} x_{8}+\frac{1}{T_{2}} u_{2} \quad \dot{x}_{9}=-\frac{1}{t_{p r}} x_{9}+\left(\frac{k_{r}}{t_{p r}}-\frac{k_{r} t_{o r}}{t_{p r}^{2}}\right) u_{1}
\end{aligned}
$$

where $x_{1}=\psi, x_{2}=d \psi / d t, x_{5}=\varphi, x_{6}=d \varphi / d t$, and $x_{3}, x_{4}, x_{7}, x_{8}, x_{9}$ denote the state variables which describe the dynamic of DC motors and the coupling effects, and $y_{h}=x_{1}$ and $y_{v}=x_{5}$ are defined as the controlled outputs. The numerical values of physical parameters of the helicopter are listed in Table 2.

State equations (4.1) can be decomposed in to two subsystems as introduced by (2.3) with $\mathbf{X}_{h}=\left[x_{1}, x_{2}, x_{3}, x_{4}\right]^{\mathrm{T}}$ and $\mathbf{X}_{v}=\left[x_{5}, x_{6}, x_{7}, x_{8}, x_{9}\right]^{\mathrm{T}}$. Such a model description is specified by

$$
\begin{aligned}
& \mathbf{A}_{h}=\left[\begin{array}{cccc}
0 & 1 & 0 & 0 \\
-\frac{\tau_{g}}{I_{\psi}} & -\frac{b_{\psi}}{I_{\psi}} & \frac{b_{1}}{I_{\psi}} & 0 \\
0 & 0 & -\frac{1}{T_{1}} & \frac{1}{T_{1}} \\
0 & 0 & 0 & -\frac{1}{T_{1}}
\end{array}\right] \quad \mathbf{A}_{v}=\left[\begin{array}{ccccc}
0 & 1 & 0 & 0 & 0 \\
0 & -\frac{b_{\varphi}}{I_{\varphi}} & \frac{b_{2}}{I_{\varphi}} & 0 & -\frac{1}{I_{\varphi}} \\
0 & 0 & -\frac{1}{T_{2}} & \frac{1}{T_{2}} & 0 \\
0 & 0 & 0 & -\frac{1}{T_{2}} & 0 \\
0 & 0 & 0 & 0 & -\frac{1}{t_{p r}}
\end{array}\right] \\
& \mathbf{B}_{h}=\left[0,0,0, \frac{1}{T_{1}}\right]^{\mathrm{T}} \quad \mathbf{B}_{v}=\left[0,0,0, \frac{1}{T_{2}}, 0\right]^{\mathrm{T}}
\end{aligned}
$$


Table 2. Physical parameters of the helicopter (Morbidi, 2006/2007)

\begin{tabular}{|c|c|c|}
\hline Parameter & Value & Units \\
\hline \hline$\tau_{g}$ & 0.0383 & {$[\mathrm{~N} \mathrm{~m}]$} \\
\hline$a_{1}$ & 0.186 & {$\left[\mathrm{~N} \mathrm{~m} /\left(\mathrm{MU}^{2}\right)\right]$} \\
\hline$b_{1}$ & -0.0445 & {$[\mathrm{~N} \mathrm{~m} /(\mathrm{MU})]$} \\
\hline$a_{2}$ & 0.033 & {$\left[\mathrm{~N} \mathrm{~m} /\left(\mathrm{MU}^{2}\right)\right]$} \\
\hline$b_{2}$ & 0.0294 & {$[\mathrm{~N} \mathrm{~m} / \mathrm{MU}]$} \\
\hline$b_{\psi}$ & 0.005 & {$\left[\mathrm{Kg} \mathrm{m}^{2} / \mathrm{s}\right]$} \\
\hline$I_{\psi}$ & 0.00437 & {$\left[\mathrm{Kg} \mathrm{m}^{2}\right]$} \\
\hline$b_{\varphi}$ & 0.00869 & {$\left[\mathrm{Kg} \mathrm{m}^{2} / \mathrm{s}\right]$} \\
\hline$I_{\varphi}$ & 0.00414 & {$\left[\mathrm{Kg} \mathrm{m}^{2}\right]$} \\
\hline$T_{1}$ & 0.1 & {$[\mathrm{~s}]$} \\
\hline$T_{2}$ & 0.25 & {$[\mathrm{~s}]$} \\
\hline$k_{r}$ & -0.00891 & {$[\mathrm{~N} \mathrm{~m} /(\mathrm{MU})]$} \\
\hline$t_{o r}$ & 2.7 & {$[\mathrm{~s}]$} \\
\hline$t_{p r}$ & 0.75 & {$[\mathrm{~s}]$} \\
\hline$k_{g y r o}$ & 0.015 & {$[\mathrm{~N} \mathrm{~m} / \mathrm{s}]$} \\
\hline
\end{tabular}

and the model uncertainties

$$
\begin{aligned}
& \Delta \mathbf{F}_{h}=\left[0, \Delta f_{h}, 0,0\right]^{\mathrm{T}} \\
& \text { with } \quad \Delta f_{h}=\frac{1}{I_{\psi}}\left[-\left(\sin \left(x_{1}\right)-x_{1}\right) \tau_{g}+a_{1}\left(x_{3}\right)^{2}-k_{g y r o} \cos \left(x_{1}\right) x_{6} u_{1}\right] \\
& \Delta \mathbf{F}_{v}=\left[0, \Delta f_{v 1}, 0,0, \Delta f_{v 2}\right]^{\mathrm{T}} \\
& \text { with } \quad \Delta f_{v 1}=\frac{1}{I_{\varphi}}\left(a_{2}\left(x_{7}\right)^{2}-\frac{k_{r} t_{o r}}{t_{p r}} u_{1}\right) \quad \Delta f_{v 2}=\left(\frac{k_{r}}{t_{p r}}-\frac{k_{r} t_{o r}}{t_{p r}^{2}}\right) u_{1}
\end{aligned}
$$

\section{Numerical simulation}

The time constants $T_{1}$ and $T_{2}$ are commonly known for CE150, i.e., the vectors $\mathbf{B}_{h}$ and $\mathbf{B}_{v}$ need not to be estimated. Using the parameter identification algorithm described in Section 2, the elements of $\mathbf{A}_{h}$ and $\mathbf{A}_{v}$ are estimated as illustrated in Figs. 4 and 5.

Now, the proposed FISC is applied to the CE150 helicopter and the performance of the controlled system is studied using MATLAB software. Let the desired reference vector be $\mathbf{X}_{d h}=\left[x_{1 d}, 0,0,0\right]^{\mathrm{T}}, \mathbf{X}_{d v}=\left[x_{2 d}, 0,0,0,0\right]^{\mathrm{T}}$, so the tracking errors are defined as

$$
\mathbf{E}_{h}=\mathbf{X}_{h}-\mathbf{X}_{d h}=\left[e_{1 h}, e_{2 h}, e_{3 h}, e_{4 h}\right]^{\mathrm{T}} \quad \mathbf{E}_{v}=\mathbf{X}_{v}-\mathbf{X}_{d v}=\left[e_{1 v}, e_{2 v}, e_{3 v}, e_{4 v}, e_{5 v}\right]^{\mathrm{T}}
$$

The sliding coefficients in the FISC are selected as $\boldsymbol{\alpha}_{1 h}=[0.7,0.14,1,1.8]$, $\boldsymbol{\alpha}_{2 h}=[0.7,0.1,0,0], \boldsymbol{\alpha}_{1 v}=[0.05,0.4,0.1,0.8,1]$ and $\boldsymbol{\alpha}_{2 v}=[0.85,1.2,0,0,0]$. The parameters used in the input fuzzy sets $w=1$ and $\Phi=0.09$ are simply determined to equally partition the suitable region.

Compared with the results by Boubertakh et al. $(2010,2012)$ and from the Ziegler-Nichols controller, the position control of the FISC-controlled CE150 helicopter has a better performance in the sense of less overshoot, faster time response and less oscillation around the desired positions, as shown in Figs. 6 and 7. In addition, the control actions of the FISC indicate that the chattering effect is eliminated. 

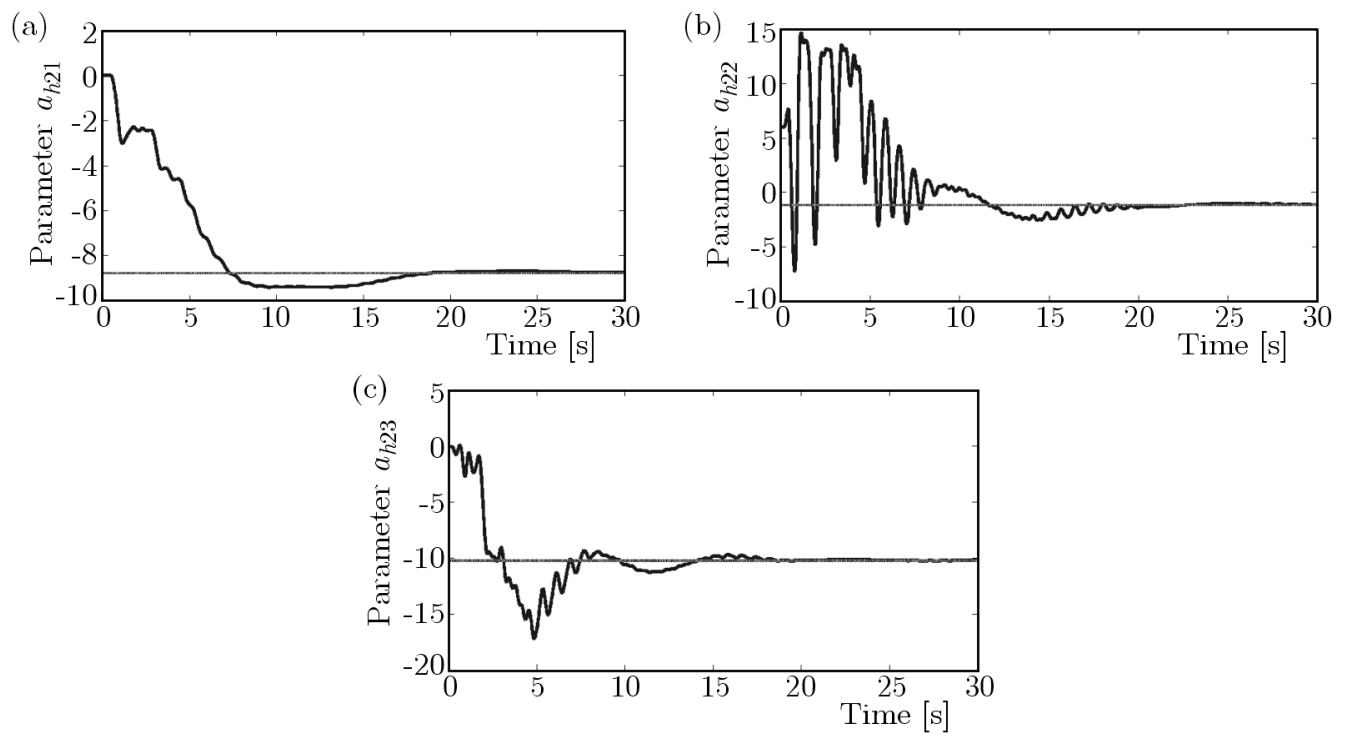

Fig. 4. Parameter convergence for (a) $a_{h 21}$, (b) $a_{h 22}$ and (c) $a_{h 23}$
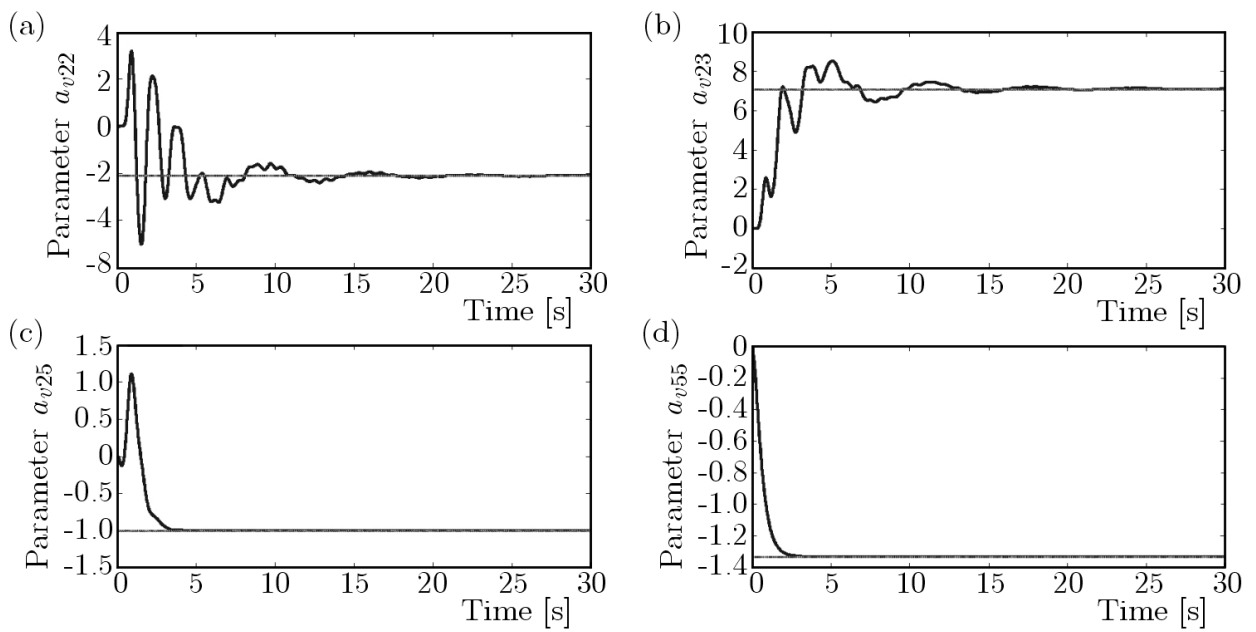

Fig. 5. Time history of parameter estimation for (a) $a_{v 22}$, (b) $a_{v 23}$, (c) $a_{v 25}$ and (d) $a_{v 55}$
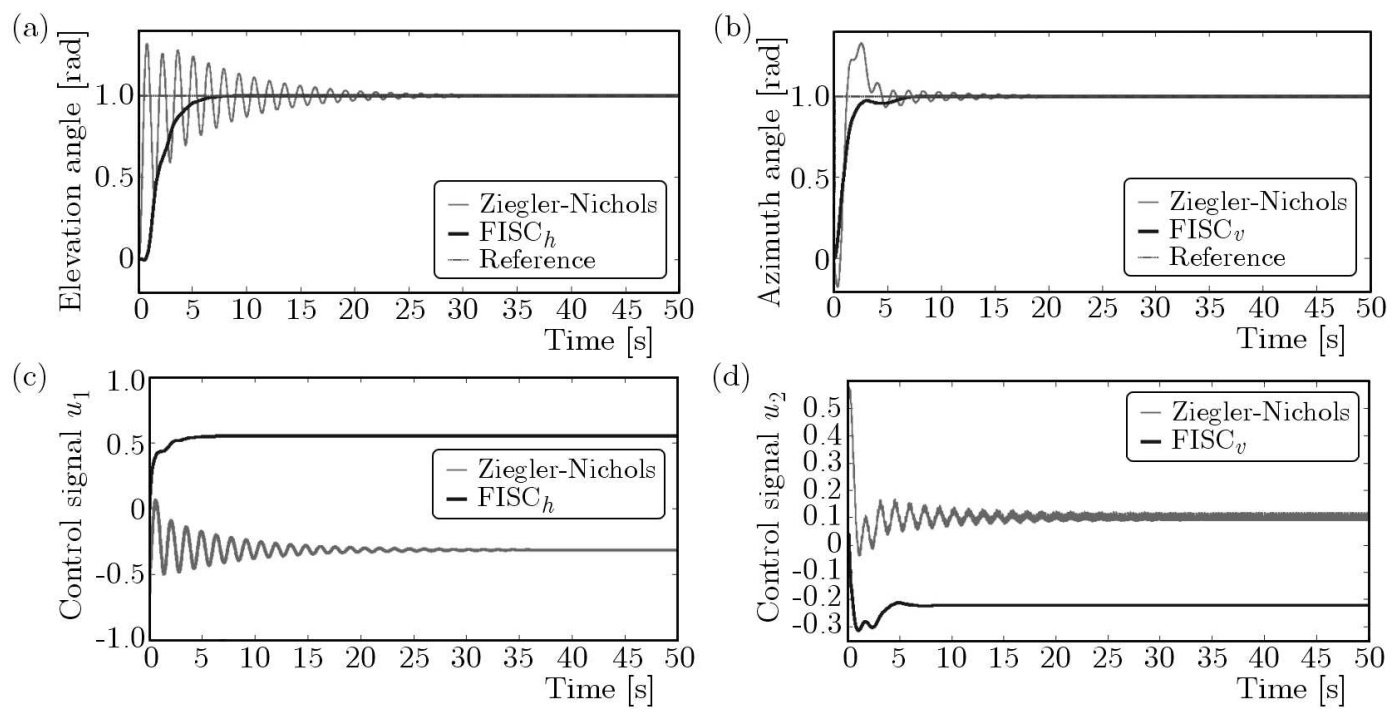

Fig. 6. Step response (a) elevation angle, (b) azimuth angle, (c) control input $u_{1}$, (d) control input $u_{2}$ 
(a)
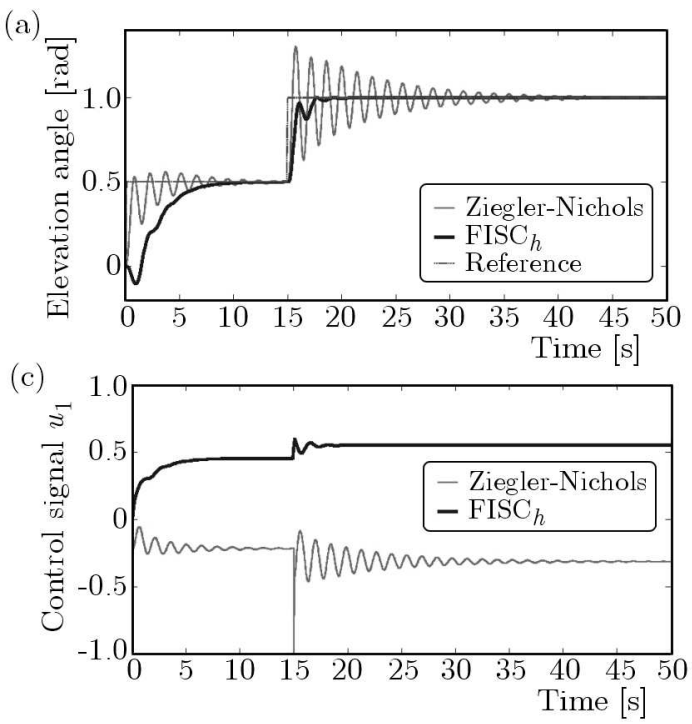

(b)

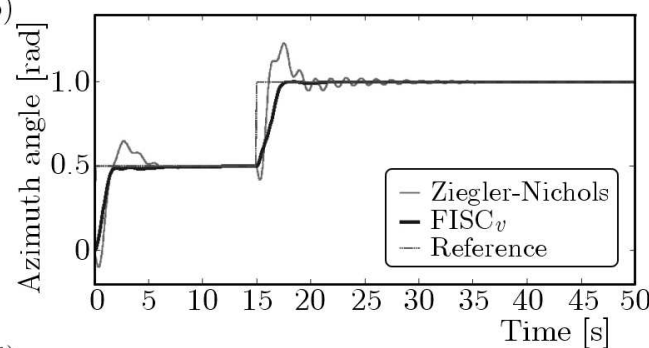

(d)

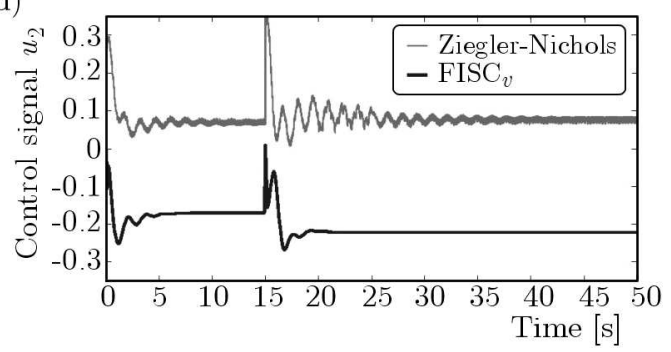

Fig. 7. Time response for tracking performance (a) elevation angle, (b) azimuth angle, (c) control effort $u_{1}$, (d) control effort $u_{2}$

In order to study the robustness properties of the designed FISC, a general structure is adopted for the external disturbance $d(t)$ as

$$
d(t)= \begin{cases}d_{1}(t) & 20 \mathrm{~s} \leqslant t \leqslant 30 \mathrm{~s} \\ 0 & \text { otherwise }\end{cases}
$$

The performance is evaluated against the abrupt and periodic disturbances by choosing $d_{1}(t)=0.4$ and $d_{1}(t)=0.4 \sin 6 t$ respectively. The tracking performance and control efforts are respectively demonstrated in Figs. 8 and 9.
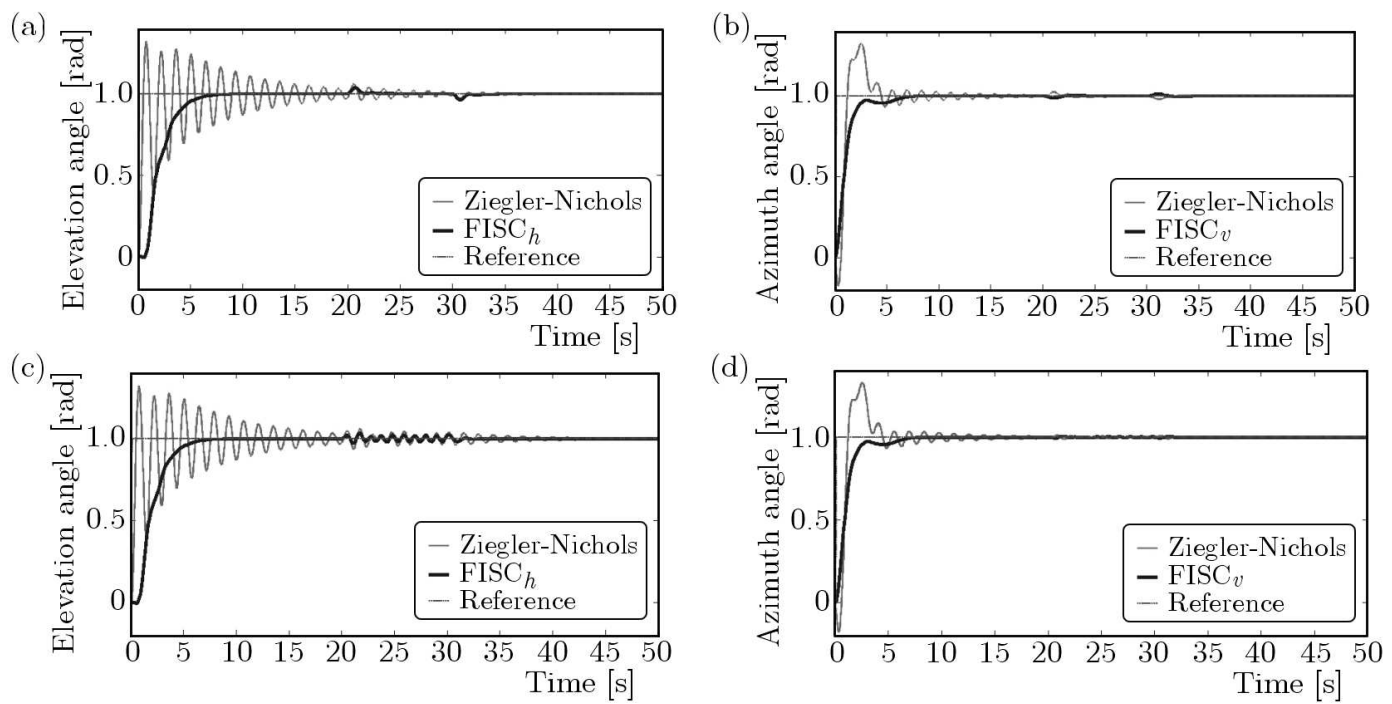

Fig. 8. Step responses in the presence of an external disturbance, (a), (b) abrupt change, (c), (d) sinusoidal disturbance 

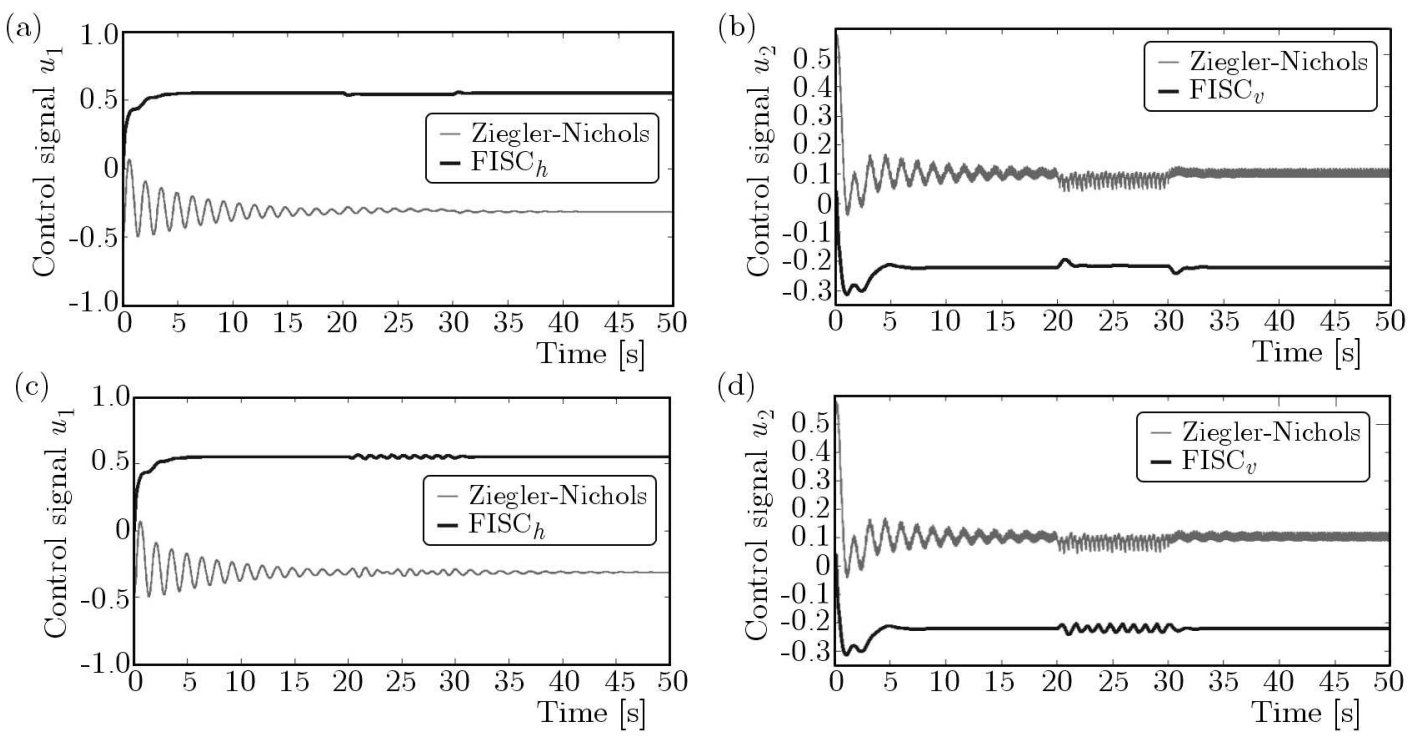

Fig. 9. Control efforts in the presence of an external disturbance, (a), (b) abrupt change, (c), (d) sinusoidal disturbance

\section{Conclusion}

In this paper, a FISC is proposed for the coupled non-linear TITO system. The cross coupling between the subsystems is considered as uncertainty. The system parameters are need not to be known exactly and may be estimated by an identification algorithm. A proportional plus integral sliding surface is used to eliminate the offset and remove the steady state error. The chattering phenomenon is alleviated by using only three Takagi-Sugeno fuzzy rules. The proposed method is then used to control a 2DOF laboratory helicopter. The simulation results show that the proposed FISC demonstrates robust tracking performance, despite the cross-coupling effects and external disturbances.

\section{References}

1. Boubertakh H., Labiod S., Tadjine M., 2012, PSO to design decentralized fuzzy PI controllers application for a helicopter, Control and Automation (MED), 2012 20th Mediterranean Conference on IEEE, 633-637

2. Boubertakh H., Tadjine M., Glorennec P.-Y., Labiod S., 2010, Tuning fuzzy PD and PI controllers using reinforcement learning, ISA Transactions, 49, 543-551

3. Chien I.-L., Huang H.-P., Yang J.-C., 1999, A simple multiloop tuning method for PID controllers with no proportional kick, Industrial and Engineering Chemistry Research, 38, 1456-1468

4. Chiu M.S., Arkun Y., 1990, Decentralized control structure selection based on integrity considerations, Industrial and Engineering Chemistry Research, 29, 369-373

5. Dutka A.S., Ordys A.W., Grimble M.J., 2003, Non-linear predictive control of 2 DOF helicopter model, Decision and Control, 2003, Proceedings 42nd IEEE Conferenceon IEEE, 4, 3954-3959

6. Glower J.S., Munighan J., 1997, Designing fuzzy controllers from a variable structures standpoint, Fuzzy Systems, IEEE Transactions, 5, 138-144

7. Huang Y., Kuo T., Way H., 2003, Robust vertical takeoff and landing aircraft control via integral sliding mode, IEE Proceedings-Control Theory and Applications, 150, 383-388

8. Humusoft, 1989, CE150 Helicopter Model User's Manual 
9. Hung J.Y., Gao W., Hung J.C., 1993, Variable structure control: a survey, IEEE Transactions on Industrial Electronics, 40, 2-22

10. Iohnnou P.A., Sun J., 1996. Robust Adaptive Control, Prentice-Hall

11. Jevtović B.T., MataušEk M.R., 2010, PID controller design of TITO system based on ideal decoupler, Journal of Process Control, 20, 869-876

12. Kim S.-W., LEe J.-J., 1995, Design of a fuzzy controller with fuzzy sliding surface, Fuzzy Sets and Systems, 71, 359-367

13. Morbidi F., 2006/2007, Analisi della stabilit'a robusta e controllo $H_{\infty}$ dell'Helicopter Simulator $C E, \mathbf{1 5 0}$

14. SAPIŃski B., 2005. Fuzzy control for MR damper in a driver's seat suspension, Journal of Theoretical and Applied Mechanics, 43, 179-201

15. Tavakoli S., Griffin I., Fleming P.J., 2006, Tuning of decentralised PI (PID) controllers for TITO processes, Control Engineering Practice, 14, 1069-1080

16. Utkin V., 1977, Variable structure systems with sliding modes, Automatic Control, IEEE Transactions, 22, 212-222

17. Utkin V.I., 1992, Sliding Modes in Control and Optimization, Springer-Verlag Berlin

18. Velagic J., Osmic N., 2010, Design and implementation of fuzzy logic controllers for helicopter elevation and azimuth controls, Control and Fault-tolerant systems (Sys Tol), 2010 Conference on IEEE, 311-316

19. Zhao C.-S., Zhu S.-J., He Q.-W., 2007. Fuzzy-PID control method for two-stage vibration isolation system, Journal of Theoretical and Applied Mechanics, 45, 171-177

20. Zorić N., Simonović A., Mitrović Z., Stupar S., 2013. Active vibration control of smart composite beams using PSO-optimized self-tuning fuzzy logic controller, Journal of Theoretical and Applied Mechanics, 51, 275-286 